of being called upon at some future period to support his opinion, under a perplexing and annoying crossexamination, in a court of law, conducted by a well feed advocate, skilled, it may be, to make the worse appear the better part, often retained for that very purpose, and whose aim and boast it is to place the member of a rival profession in a false and degrading position. Upon what grounds, then, can it for one moment be questioned that the medical practitioner is entitled to receive adequate remuneration for any statements or opinions he may be induced under such circumstances to advance. The only question is, whether he is to look for that remuneration from his patient, whom he may seriously offend by giving a conscientious statement, or from the office, which benefits by the integrity with which he acts, and by the intelligence which he manifests in complying with their requirements. Until, however, the Association is prepared to give a decided and influential opinion on this point, it is better that the example of the Exeter meeting should be followed. When the question shall have been fully discussed, and something like unanimity on one or other side attained, then the voice of the Association will be expressed with authority, and will, we doubt not, prove influential with its members generally, in inducing them to follow up in practice the line of conduct so marked.

We can scarcely close these observations without expressing our gratification at the successful result of this meeting, and at the high character which the Association continues to maintain throughout the provinces. Remotely situated as Exeter is from the districts in which the operations of the Association have hitherto been chiefly carried on, it might have been supposed that some falling off in the attendance of its friends-some diminution in the assembled numbers-would have been experienced; but we find that no such falling off-no such diminution-was perceptible. The Exeter meeting, like the meetings held on former anniversaries, and in more central, and therefore more accessible, places, has contributed its full share in carrying forward the Association on the full tide of prosperity, and in giving effect to those objects for which this noble institution was originally formed. In the opinion of some of its oldest and firmest friends the Association has derived increased stability from its late sojourn in this capital of the far west, and has acquired an increase of influence from the support which it has there received. The numerous and highly respectable requisition for the next meeting, and the connection which the Association acquires at Leeds with the illustrious name of Hey, afford prospects equally bright for the future; and most sincerely do we trust that the usefulness, which has hitherto marked the honorable career of the Association, will continue to characterise all its proceedings, and to gain for those who may have the management of its extended concerns the approbation of the many wise and eminent men who are enrolled among its ranks.

\section{UNIVERSITY OF EDINBURGH.}

After an uninterrupted service of forty-four years, Dr. Home has resigned the chair of practice of physic in the University of Edinburgh. Dr. Alison has been unanimously chosen by the town-council to fill his place. The chair of theory of medicine, thus vacant, will be filled by election on the 20th of September next. On retiring from his professorship, Dr. Home stipulated for an annuity of $£ 300$ per annum; one half of this sum is to be taken from the professor of the practice of physic; the other half from the professor of the theory of physic.

The chair of pathology, vacant by the resignation of Dr. Thomson, has been filled by the election of Dr. Henderson. The candidates were Dr. Cragie, Dr. Handyside, and Dr. Henderson. On the first ballot, Dr. Cragie obtained 12 votes; Dr. Henderson 11 ; and Dr. Handyside 10 . The name of the latter gentleman was then struck off the roll and a new ballot taken, when Dr. Henderson obtained 17 votes, and Dr. Craigie 16. The rejection of so distinguished a pathologist as Dr. Cragie appeared to excite considerable discontent amongst many members of the council.

\section{EXPERIMENTAL} AND

\section{PRACTICAL RESEARCHES} ON THE

\section{NATURE AND ORIGIN OF TUBERCLES IN THE LUNGS.*}

\section{By William Adnison, Esq., F.L.S., Malvern.}

The author commences by saying that blood corpuscles examined by the microscope exhibit the appearance of two circles, one within the other. When the corpuscles are crowded together, they become elongated or compressed into a variety of shapes, and the outline of the inner circle is quite as much altered by pressure as that of the outer; but the matter or substance within the inner circle is never seen to mingle with that of the outer one, and when the pressure is removed, both circles regain their pristine form; hence, blood corpuscles consist of two soft elastic vesicles-the one being central within the other-and they possess the following structure :-

1. A highly elastic tunic, forming the outer and larger vesicle.

2. A similar tunic, forming the inner and smaller vesicle.

3. A coloring matter which fills the outer vesicle, occupying the space between the two tunics; and

- Abstract of a paper read at the anniversary meeting of the Provincial Association, August 3, 1842. 
4. A peculiar, organisable colorless mater filling the inner vesicle.

In all classes of beings, the blood corpuscles-as far as the author's expcrience extends-have the same kind of structure. In every drop of human blood, and in that of other mammalia, however minute, there are two very distinct kinds of corpuscles - the one red, the other colorless-and they may always be discriminated, without the application of any extrancous substance, by the following experiment:-

A spot of recent blood, just withdrawn by a puncture at the back of the hand, was pressed into a very thin film between two slips of glass, the surfaces of which fitted evenly on each other, and examined by the microscope. During the examination, the glasses were frequently pressed together gently with the point of a finger, so as to put the corpuscles in motion; by these means the eye was directed to several remarkable corpuscles, which appeared harder or of a firmer structure than the rest; for the softer corpuscles, urged by the gentle motions given to the glass, passed to and fro around them, losing their figure. The softer corpuscles adhered together in rows; but these had no such disposition.

The corpuscles thus discriminated werc generally found to be larger than the others,-the inner vesicle was larger, and numerous minute dark points, or molecules, were distinctly visible within it. By gently altering the focus of the microscope to and fro, they presented a peculiar and characteristic pearly aspect, and exhibited refractions of the light, which could not be produced by the same means in the softer corpuscles. These are the colorless corpuscles of the blood.*

By repeating the foregoing experiment with blood just drawn from any inflamed surface-as a pimple or the base of a boil-they are found very abundantly, and in blood taken from the skin of a patient in scarlet fever they are remarkably numerous and conspicuous.

It is impossible to form an accurate estimate of the depth or tone of the color of the red corpuscles, viewed singly as microscopic objects; hence the distinctive character of the colorless blood corpuscle, seen in this way, does not arise so much from any striking or obvious difference of color as it does from its greater resistance to pressure-from the greater relative size of the inner vesicle-from the dark points or molecules visible within it-and from the peculiar manner in which it refracts the light.

The author remarks, that a black spot on a white ground, having a diameter of only $\frac{1}{500}$ of an inch, is distinctly visible to a person with a good sight when held at the shortest distance for distinct vision. There are objects, not only in the blood, but in all organised tissues, visible as similar black points, when magnified 250 diameters; hence, their actual measure cannot be greater than 100000 of an inch; to these objects he applies the term molecules. There are, he says, other objects in the blood and in all organised tissues, which, with the same magnifying power, are visible as bright pellucid points bounded by a strongly defined dark circle; these he calls granules-terms having no reference whatever to the actual nature of the objects. Molecules become granules, granuules form

" London Medical Gazette," December, 1840, January, 1841, and April, $19+2$. vesicles or corpuscles, and vesicles become cells. The mean diameter of the human blood corpuscle may be taken at $\frac{1}{2800}$ of an inch, and it takes four or five granules to reach across the diameter of a corpuscle; hence, the measure of a granule cannot be greater than from ${ }_{11000}$ to ${ }_{15}$ boo of an inch. Granules, however, vary considerably in size, and no precise measurement can be adapted to them. Molecules are the same objects, called by Dr. Martin Barry, in his memoirs on the corpuscles of the blood," "dark objects," " dark globules," and "foundations of new cells." Granules are synonymous with the "discs" of the same author.

After noticing the various appearances presented by the red corpuscles, and having stated that the colorless corpuscles are most readily obtained for microscopical examination from the buffy coat of inflammatory blood before it coagulates, the author remarks that the elements of the blood visible by the microscope, without any manipulation, are-

Jst, The red corpuscles; 2nd, the colorless corpuscles; $3 \mathrm{rd}$,molecules and granules in the interior of the colorless corpuscles; 4 th, similar molecules and granules isolated in the liquor sanguinis, and independent of the corpuscles; 5 th, the fibrine, which may be seen by the microscope coagulating in the form of delicate cylindrical fibres, having a diameter even less than that of the molecules.

The fibrine does not form globules or corpuscles of any kind. Molecules, granules, and colorless corpuscles, enveloped in a dense net-work of fibrinous fibres or filaments, with variable quantities of entangled serum form the entire mass of the buffy coat of the blood.

The paper then relates at considerable length the eflects of water, the dilute acetic acid, and the liquor potassæ upon the red and colorless corpuscles of the blood, and on pus corpuscles.

The effects of water on the red corpuscles varies exceedingly in different experiments, apparently with the length of time the corpuscles have been taken from the vessels, and with the proportions in which the water is applied. It sometimes renders the corpuscles smaller-one half their previous size-without dissolving or rupturing any of their structures; or it dissolves the outer tunic, disperses the eoloring matter, and leaves the inner vesicle faintly visible. Sometimes the corpuscles imbibe the water, especially if a little gum be previously dissolved in it, and they grow very large by imbibition. The colorless corpuscles do not experience any sudden change on the addition of water, but they slowly imbibe the fluid; the molecules and granules in their interior become more and more distinct, and at the end of an hour or two the corpuscles, after swelling considerably, burst open and discharge their contents.

The effect of the dilute acetic acid on the blood corpuscles is similar to that of water; it acts more energetically; frequently after the application of the acid the outer vesicle of the red corpuscles is rendered very faint, but not destroyed - the color disappears, but the corpuscle retains its circular figure-it is much enlarged by imbibition, and the inner vesicle is very distinct. $\dagger$ The

- Philosophical Transactions for 1841, and 1842.

+ These experiments corroborate the result of those of Dr. Rees and Mr. Lane,-Guy's Hospital Reports. 
colorless corpuscles imbibe the dilute acid, and molecules and granules may be seen within them in motion. After a few hours, or sooner, the corpuscles burst, and discharge their contents.

The phenomena displayed on the addition of liquor potassæ (Brandish's alkali) to the blood corpuscles are very remarkable; the first effects of the fluid are very rapid and energetic; after a short time, by following the directions given in the paper, the alkali may be observed slowly diffusing itself among the corpuscles. The red corpuscles may be seen to explode and disappear instantaneously, or to burst open gradually, and discharge their contents, and many singular changes of form may be witnessed. The colorless corpuscles may be completely and slowly dissected by a proper application of the liquor potassæ-all the stages of the process and the number and nature of their contents may be readily seen by the microscope. For this purpose, a drop of the liquor sanguinis from the surface of buffy blood is removed by the point of the finger, and transferred to a slip of glass, previous to coagulation; it should be spread out a little on the glass, and the liquor potassa cautiously added from the point of a penknife or a lancet, while the corpuscles, without any covering, are under examination. This manipulation, with a little practice, may be readily accomplished with one of the small, upright French microscopes, and by moving the slip of glass gradually forward, the progress of the alkali may be watched for some time. Rapid changes take place; molecules and granules are developed in the interior of the corcorpuscles, which enlarge by imbibition, and then dehisce or explode, the molecules and granules pouring out of them in great numbers; if the alkali be acting weakly, the molecules swell to granules, and the granules enlarge previous to their final disappearance by dissolution in the liquid.

A remarkable alte ration may be sometimes witnessed in the corpuscles, which throws some light on their future development into cells or granulated vesicles; they give a sudden jerk, and at the same instant enlarge to twice or three times their former dimensions, without losing their circular outline. The molecules and granules' in their interior are more widely separated from each other, but not dispersed; they are seen withiu the enlarged corpuscle connected together by delicate filaments; the corpuscles. in this state exactly resemble, in structure and appearance, the cells of many vegetable tissues. The isolated or independent molecules and granules visible by the microscope, in the buffy coat of the blood before coan gulation, are similarly acted on by the liquor potassathey become greatly agitated when the alkali reaches them, enlarge by imbibition, : and ultimately disappear.

Pus corpuscles differ in no respect from the colorless blood corpuscles, except only as they are altered in size and appearance by exhaling their own contents or by imbibing the fluids with which they are associated. Water and the dilute acetic acid cause them to enlarge, developing the molecules and granules in their interior. The effects of the liquor potassæ upon them are very singular and instructive. On the application of this liquid the corpuscles immediately begin to enlarge by imbibition; a great number of granules become exseedingly conspicuous within them ; finally, they burst open or explode, and the granules are dispersed around.

Several cases and experiments are minutely de. tailed in corroboration of the foregoing statements, from which the two following are selected :-

CASE V.-Shingles.-One of the clear transparent vesicles was opened with the point of a lancet, and a great number of colorless blood corpuscles was found by the microscope in the fluid. Many of them exactly resembled the corpuscles in the blood; others appeared a little shrivelled, and a few were remarkably large. All of them contained molecules; the large ones had several bright granules, which, on close inspection, were evidently moving within them-that is, the granules disappeared in one part of the corpuscle, and became visible in another. This was repeated several times during the observation, apparently from the working ("writhing") or undulation of a fluid, which, by alternately dividing and coalescing, and then again dividing, formed the granules, which were sometimes visible at one spot, and then at another. The author has seen similar phenomena in the inner vesicle of the red corpuscles after the ad. dition of a drop of gum water.

A little water was now added to the corpuscles from the point of a lancet; after a few minutes the shrivelled corpuscles became quite plump. Liquor potassæ was afterwards added very cautiously in the same way, and the corpuscles, one after another, dehisced. At first they gave a sudden jerk, and en. larged to twice or three times their former dimensions, displaying the granules in their interior; and then they burst open by a kind of explosion, each corpuscle discharging about twelve large granules, which weré gradually dissolved.

Case.-Troublesome Catarrh (Hay-fever).-Copious discharge of clear transparent fluid from the nostrils; "a perfectly limpid drop of the mucus was examined by the microscope, and I was somewhat surprised to find in it a great number of colorless corpuscles, resembling the colorless corpuscles of the blood. There were, indeed, corpuscles of all sorts, forming a complete series, with gradual transitions, from the colorless blood corpuscles through all the forms and varieties of pus corpuscles, up to large, round, granulated córpuscles and epithelium cylinders. My astonishment was great when I found most of these corpuscles provided with vibratile cilia in the most active state, so that some of the corpuscles were actually moving about by their means; and not only were the cilia in motion, but the molecules and granules in the interior of the corpuscles were in a very active state; they could be seen rapidly shifting their position within the corpuscles.

I examined no less than six different specimens of the mucus from the same individual, and I always saw a series of similar objects.

CASE VII.-Cancer of the Breast (existing some years).-Arm œdematous; the back of the hand had been punctured some weeks ago to relieve the swelling, and a serous fluid has been discharged from it ever since. The author frequently examined this fluid by the microscope, and always found in it a flbrinous coagulum, and corpuscles resembling in every particular the colorless corpuscles of the blood, some. what altered by shrivelling. When the arm was cool 
and free from pain, the fluid discharged was perfectly limpid, as colorless as spring water, but corpuscles were found in it; and after standing ten or twelve hours, a delicate but almost invisible net-work of fibrine could be drawn out of it with the point of a needle. Sometimes the arm, without any obvious cause, became much more red, swollen, and painful, and then the discharged fluid had the appearance' of ordinary serum; the number of corpuscles was much increased, and the coagulum was more dense. On the addition of water the corpuscles swelled out and enlarged; with liquor potassæ they burst open, discharging large granules. From the result of this and other experiments, it is evident that the serous fluids effused in dropsies differ from the serum after venesection; they contain fibrine and corpuscles. The fluid at the surface of inflammatory blood is the liquor sanguinis, highly charged with molecules, granules, and colorless corpuscles; it is a strong solution of fibrine. The limpid fluids discharged by œdematous swellings are of a precisely similar nature, with a less quantity of fibrine and a less number of corpuscles; the varieties of coagulable lymph are intermediate between the two extremes.

The conclusions which the author draws from his observations and experiments are,-

1. The blood at all times contains two very distinct kinds of corpuscle, the red and the colorless.

The red corpuscles are in progressive stages of development, during which they gradually lose color, the inner vesicle increasing in size at the expense of the coloring matter of the outer one, the colorless corpuscle being the mature inner vesicle (or, as it is termed, the nucleus) of the red corpuscle.

[It is here remarked, that the nucleus is the nutrimental organ of the wall or membrane of a cell, and that the inner vesicle of a blood corpuscule is not a nucleus.]

2. Blood corpuscles possess the function of imbibition, and have the power of choosing or selecting the elements they imbibe, and consequently the power of separating what they require from the materials by which they are surrounded, and of re-combining the imbibed elements to form new products. By the exercise of these functions on the atmosphere, during their passage through the lungs, the red corpuscles change their color, and exhale those elements which are not required, or would be useless in their greater circulation through the body.

3. All abnormal discharges, and all the varieties of serum and coagulable lymph, are modifications of the liquor sanguinis - stronger or weaker solutions of fibrine. All the varieties of pus and lymph corpuscles are more or less altered colorless blood corpuscles-altered either by imbibing and growing larger, or by exhaling, shrivelling, and becoming less. In fact, all abnormal products are effusions, and not secretions.

In order to illustrate and confirm the doctrine contained in the last of these conclusions, the author details several observations on the inflamed web of the frog's foot, from which it appears that the corpuscles, called "lymph globules," well known to all microscopical observers, accumulate in a very singular manner, and to an extraordinary amount, in the inflamed vessels. In many of these vessels they were so numerously congregated, that the whole interior seemed to be lined with them. The current of the red corpuscles passed by them, keeping in the centre of the vessels, and leaving a well-marked interval between the red current in the centre and $t$ e boundary of the vessels, which was entirely occupied by "lymph globules."

A great many of these lymph globules or corpuscles are stated to have been seen exterior to the line marking the outline of the vessels, and several similar corpuscles were distributed on the intervening tissue. These results of inflammation were observed not in one only, but in all the experiments. The stimulant chiefly used was water, varying in temperature from $95^{\circ}$ to $110^{\circ}$. The phenomena, witnessed by the author, appeared to him to corroborate the opinion already entertained by some very distinguished anatomists and physiologists, that the minute capillary distributions of the blood run in channels in the tissue, and that there are no membranous tubular coats separating the currents of the blood from the structure. The slightest violence or injury immediately determines the colorless corpuscles to the part, which arrange themselves along the outline or boundary of the channel.

Experiments were made on the blood corpuscles of the frog, in order to determine the nature of the "lymph globule." Water, the dilute acetic acid, and especially the liquor potassa, were used for this purpose, and the effects produced are described in the paper. The author infers from them that the "lymph globule" of the frog is the colorless blood corpuscle, or the mature inner vesicle of the red corpuscle. It discharges, under the influence of liquor potassæ, a great multitude (at least fifty) of molecules and granules. The essential character of "vital turgescence" (the "modelling process" of Macartney) is a normal accumulation of the colorless blood corpuscles; for the purposes of growth, nutrition, or reparation, and where there is an active and extensive "vital turgescence," the blood indicates it, as in pregnancy. Inflammation is an abnormal and more or less excessive "vital turgescence," and the colorless corpuscles in excess form pus corpuscles.

The cells of vegetable tissue, the colored cells in the petals of various flowers, the structure of the pollen grain and of the polygastric animalcules, are minutely examined and described. Cells have the following structure :-

1. A transparent membrane, forming the cell wall.

2. The nucleus, or nutrimental organ of the cell wall, and usually attached to it.

3. Molecules and granules, of various sizes, within the cell.

4. Molecules and granules within the nucleus.

The size of the cell, the thickness of its wall, and the development of the nucleus, have no especial reference to the peculiar sensible qualities of the matter secreted by the cell. In the cultivated fruits-the cherry and the strawberry-the cells are inordinately enlarged, their walls thick, and the nucleus (with its molecules and granules) attached to the wall, is very conspicuous; but the fluid distending the cells is much blander than in the natural condition of these fruits, and comparatively tasteless. The granules of the cells are small and inconspicuous. But, on the other hand, the granules of the cell have a develop- 
ment corresponding with the peculiar sensible qualities of the secretion, of which the fleshy leaves of sedum acre afford excellent examples. This plant flourishes on the bare rock, exposed to a burning sun; yet the leaves are very juicy (evincing an acti. vity of function), and have very strong sensible qualitios. The membrane or wall of the cells is thin, the nuoleus indistinct, but the granules of the cell are very numerous and large.

In a parti-colored petal, each cell may be seen to possess its own color, without any intermixture with that of the adjoining ones. Cells with a purple or red pigment are found in juxta-position with others of a brilliant yellow, and these are intermingled with cells devoid of color. In the secreting vesicles of the liver the wall is thin, and so extremely tender that it will hardly bear any examination. There is no apparent nucleus, but the molecules and granules within the vesicle are numerous and conspicuous.

The foregoing experiments (here briefly stated) are brought forward to strengthen the conclusion which it is the author's object to establish-viz., that all secretions take place in the interior of granulated vesicles or cells, not by transudation from one tube (a blood vessel) into another (a duct); consequently that "tubercles in the lungs," "tubercular infiltrations," "hepatisation," and "pus," are not secreted products, but simply the elements of the blood effused by an excessive "vital turgescence" (or inflammatory action), having their peculiar characters determined by the texture and function of the structure, and by the amount or activity of the turgescence.*

A singular fact is stated with regard to the animalcules; it may be witnessed in all of them by the application of liquor potassæ (which the author calls his dissecting fluid). ' It penetrates the transpurent tunic composing the body of the animalcule, by imbibition, and soon causes it to burst open or explode, precisely in the same way as the colorless blood or pus corpuscle; and the so-called stomachs of the creature are forcibly discharged, or thrown out one after another, thus becoming objects for minute microscopical scrutiny. The stomachs (?) swell and burst in like manner, precisely as the granules discharged from the lymph corpuscles of the frog, or from the pus corpuscle. 'These stomachs the author believes to be granulated vesicles, performing their functions by imbibition; and not by assimilating or digesting food, voluntarily taken." The vital powers of the annimalcules are totally inefficient in opposing the imbibition of the poison, and their stomachs may be seen enlarging in the' interior of the body prior to the rupture of the integument.

The paper (which will be published in the forthcoming volume of the "Transactions" of the Association) concludes with researches on the nature of tubercles of the lungs.

- The author refers to Mr. Goodsir's paper, and corroborates his opinion with respect to secretion.

\section{STAMMERING.}

An operation for the cure of staminering by $M$. Velpeau, recently terminated fatally, in consequence of purulent absorption. We believe that the operation has long since been abandoned by $M$. Dieffenbach,
ON THE

\section{E F F I C A C Y OF I N DIA N H E M P IN}

\section{SOME CONVULSIVE DISORDERS.}

By W. LeY, M.R.C.S., London.

[Read at the Exeter Meeting of the Provincial Medical and Surgical Association.]

The "British and Foreign Review" for July, 1840, notices a pamphlet by Dr. O'Shaughnessy, professor of chemistry in the Medical College, Calcutta, in which he strongly recommends the use of the resin of the garden hemp as a narcotic and antispasmodic. As the pamphlet is not to be had in this country, I can only refer to the accounts given of it in our reviews.

His success in relieving the spasms in tetanus and hydrophobia, although he was not successful in the cure of his patients, made me very desirous of using the ymedy for the relief of the patient, whose history I will now detail.

A lady had some years previously reccived a shock and a blow by falling backwards on a tree, supposing that she was sitting down on a chair or stool behind her. The immediate consequences were not very severe, but after months of ill health, she was considered to have spinal disease, and at the time I speak of, had been confined to Dr. Arnott's hydrostatic bed about five years. This confinement was attended with much suffering, as the Indian-rubber sheet, which covers the water, will not last more than six or eight months, and the patient must be moved to another bed. The motion from one bed to another produces great pain, which shoots up the 'spine, and is followed usually at night by spasms of the muscles of the back; the latter are so violent as to draw the body into the form of an arch ; this spasm continuing about a miuute, is followed by a relaxation of the muscles, so that the patient cannot raise her head, and an expiration broken by sobs, and cough . with a sense of choking, ensues; the shooting pains in the back continue, with intermissions of a few seconds only, and with intervals of from two to three minutes the spasms return, and continue for a period of seven or eight hours, until the severity of exhaustion seems to produce a sleep, broken by occasional spasm, and the continued shoot. ing pain in the back. 'A day of exhaustion nearly amounting to unconsciousness, with faintness, cough, sickness, and the constant aching pain in the muscles of the neck and back, produced by straining from spasms, and maintaining the sensation of choking, is followed by a return of the spasms at night.

I ordered a grain and a half of the extract of hemp every half hour; after the fifth dose my patient felt the effects powerfully; she complained that she - was sure she had taken too much; the muscles became relaxed, and she fell into a.tranquil, but overpowering sleep of ten hours' duration; she then awoke with little exhaustion, little of the aching pain in the neck, and pleased beyond expression at the relief she had received; the shooting pains in the back, however, continued, and at night the spasms retiirned; still she feared to take as many pills as would produce any very decided effect " but her sufferings were much moderated, and the duration of the disease proportionally shortened; the spinal irritation had produced a contraction of tho muscles of the left leg; the foot was 